\title{
Publisher pulls 58 articles by Iranian scientists over authorship manipulation
}

\section{Cull of papers follows similar discoveries in 2015.}

\section{Ewen Callaway}

01 November 2016 I Clarified: 03 November 2016

A tranche of 58 articles authored by 282 Iran-based researchers were retracted today by a leading scientific publisher, which said it had found signs that the peer review and publication processes had been compromised.

BioMed Central (BMC) will retract 28 articles and investigate another 40, whereas Springer will pull 30 papers and investigate another 9. Both organizations are run by Nature's publisher, Springer Nature. (Nature's news and comment team is editorially independent of the publisher).

The actions follow an investigation, prompted by a whistleblower, that found "evidence of plagiarism, peer review and authorship manipulation, suggestive of attempts to subvert the peer review and publication system to inappropriately obtain or allocate authorship," according to a press release.

All the papers that Springer is retracting showed evidence of authorship manipulation and peer-review manipulation, and $70 \%$ showed evidence of plagiarism. For the BMC papers, all showed evidence of authorship manipulation, $57 \%$ showed evidence of peer-review manipulation and $93 \%$ showed evidence of plagiarism, says BMC spokeswoman Amy Bourke-Waite.

Last year, Springer retracted 64 articles over concerns that they had been compromised by 'fake peer-review' schemes, in which fabricated peer-review reports were submitted from bogus e-mail addresses linked to the names of real scientists, and BMC withdrew another 43 for similar reasons earlier in 2015. Several other major publishers have reported similar discoveries of fake peer review.

Both organizations said they would clamp down on fake peer review after those incidents, and BMC ended the practice of allowing researchers to directly suggest potential reviewers in its submission system. Now, such suggestions mostly come via a cover letter, with evidence of the potential reviewer's authenticity, says Bourke-Waite. BMC has also made it more difficult to alter the authorship of papers during the publication process, she says.

The authors of the papers have been notified and some have responded, according to Bourke-Waite. The retractions appear in three Springer journals - one in Comparative Clinical Pathology, four in the Journal of Parasitic Diseases, and 25 in Tumour Biology - and four BMC journals, including 23 papers in Diagnostic Pathology, two each in Cancer Cell International and the Journal of Ovarian Research and one in the World Journal of Surgical Oncology.

Nature | doi:10.1038/nature.2016.20916

\section{Clarifications}

Clarified:This article has been amended to clarify that Nature's news and comment team is editorially independent of its publisher, Springer Nature. 